\title{
Sharing vesicles between central presynaptic terminals: implications for synaptic function
}

\author{
Kevin Staras ${ }^{1 *}$ and Tiago Branco ${ }^{2,3}$ \\ School of Life Sciences, University of Sussex, Brighton, UK \\ 2 Wolfson Institute for Biomedical Research, University College London, London, UK \\ ${ }^{3}$ Department of Neuroscience, Physiology and Pharmacology, University College London, London, UK
}

\section{Edited by:}

Robert Renden, Heidelberg University,

Germany; Union chimique belge

Pharma SA, Belgium

\section{Reviewed by:}

Daniel Gitler, Ben-Gurion University of

the Negev, Israel;

Lucas Pozzo-Miller, University of

Alabama at Birmingham, USA

${ }^{*}$ Correspondence:

Kevin Staras, School of Life Sciences,

University of Sussex, Brighton, BN1

90G, East Sussex, UK.

e-mail:k.staras@sussex.ac.uk
Presynaptic terminals in hippocampal neurons house functionally distinct vesicle pools, the size, structure and biochemical features of which are major determinants of presynaptic strength and performance. In classical models of synaptic function these vesicle pools are synapse-specific, but accumulating evidence is now demonstrating that some vesicles are laterally mobile along axons and readily shared in a functional manner across adjacent terminals. In effect then, these mobile vesicles represent a further class of synapse-spanning vesicle pool, or "superpool". Here we outline the characteristics of this additional pool type, discussing its structural organization within axons and presynaptic terminals as well as its relationship with conventional vesicle pools. We draw comparisons between extrasynaptic vesicle dynamics and the growing literature on extrasynaptic mobility of non-vesicular synaptic elements which, taken together, raise important questions about the operational independence of adjacent release sites. We also examine the functional implications of lateral vesicle sharing, from the notion that extrasynaptic vesicles can contribute to the release capabilities of individual terminals, to its potential role as a substrate for facilitating changes in synaptic weight as a basis for plasticity.

Keywords: synaptic vesicle, presynaptic terminal, neuron, synaptic transmission, imaging

\section{BACKGROUND}

Ultrastructural work has revealed the key anatomical characteristics of presynaptic terminals. Perhaps the most striking feature of individual boutons observed in electron micrographs is the cluster of synaptic vesicles (SVs), first described by De Robertis and Bennett $(1954,1955)$ and Palay (1956), lying close to a specialized area of the membrane called the active zone and apposed to the postsynaptic structures. Although they are morphologically indistinct, SVs at a presynaptic terminal are organized into sub-categories or "pools" with functionally-defined roles (Sudhof, 2004; Rizzoli and Betz, 2005 , see other articles in this special topic). In the conventional view of synaptic organization these pools are synapse-specific in that they are operationally "coupled" to a given synaptic terminal, achieved by efficient and well-characterized recycling mechanisms which allow reuse of the same vesicle at the same synapse (Ceccarelli et al., 1973; Heuser and Reese, 1973; reviewed in Fernandez-Alfonso and Ryan, 2006). Nonetheless, recent studies, particularly in hippocampal neurons, have started to challenge this view of compartmentalized synaptic function by revealing a more labile feature of synapses - the capability for some populations of SVs to traffic along axons between different presynaptic terminals (Krueger et al., 2003; Chen et al., 2008; Darcy et al., 2006a; Fernandez-Alfonso and Ryan, 2008; Westphal et al., 2008; Staras et al., 2010). This trafficking occurs at a relatively high rate, providing a substantial turnover of vesicles at individual synapses. Moreover, many of these vesicles appear to be functional and, once mobilized, can contribute to vesicle fusion at new and spatially remote synaptic hosts. This lateral mobility offers a new view of presynaptic organization in which synapses can be functionally coupled via shared populations of fusion-competent vesicles. Here we review the main literature on vesicle sharing and consider the possible implications of this phenomenon for the operation of synapses.

\section{LATERAL MOBILITY OF SYNAPTIC VESICLES}

A clear understanding of the organization and function of individual presynaptic terminals in hippocampal neurons has been achieved in the last two decades thanks in large part to the development of sophisticated imaging technologies and synapse-specific optical probes (Ryan, 2001). These approaches permit targeted labeling of functional vesicles in synapses and, in combination with high-resolution imaging methods, have provided a detailed characterization of vesicle fusion and recycling kinetics during transmission at individual terminals (Ryan et al., 1996; Schweizer and Ryan, 2006). Using the same imaging tools, researchers have also studied the mobility of SVs. Elegant work by a number of groups has specifically examined the dynamics of vesicle movement within synapses during rest and activity (Kraszewski et al., 1996; Jordan et al., 2005; Lemke and Klingauf, 2005; Shtrahman et al., 2005). Experiments have also revealed that vesicle movement can extend beyond the conventional boundaries of the synapse. For example, trafficking vesicles in developing axons have suggested a potential role for extrasynaptic vesicle mobility in nascent synapse formation (Matteoli et al., 1992; Ahmari et al., 2000; Hopf et al., 2002; Sabo et al., 2006). Moreover, experimental evidence has also emerged to show that nominally mature neurons exhibit vesicle movement along axons between synapses (Krueger et al., 2003; Darcy et al., 2006a), raising the possibility that this process may also be func- 
tionally relevant for mature neuronal signaling. In hippocampal axons, presynaptic terminals typically form en passant boutons (Westrum and Blackstad, 1962; Sorra and Harris, 1993) that are separated from synaptic neighbors by only short $(\sim 3-7 \mu \mathrm{m}$, Ishizuka et al., 1990; Sorra and Harris, 1993; Staras et al., 2010) lengths of axon (Figure 1A) suggesting that such lateral mobility of vesicles could be important for interactions between presynaptic terminals. Subsequent studies, some employing state-ofthe-art methodological approaches such as diffraction-unlimited microscopy (Westphal et al., 2008), fluorescent nanoparticles (Zhang et al., 2009) and novel photoswitchable SV markers (Staras et al., 2010) have extended the characterization of these laterally mobile, providing further understanding of their origins and fates. Evidence suggests that trafficking vesicles can arise from stable terminals (Krueger et al., 2003; Darcy et al., 2006a; Chen et al., 2008; Westphal et al., 2008; Staras et al., 2010) and also move to and become incorporated into new synaptic hosts (Darcy et al., 2006a; Staras et al., 2010) with a turnover rate of $>4 \%$ of the total vesicle pool at a synapse (Staras et al., 2010) or higher (Westphal et al., 2008). Experiments that have tracked the fate of vesicles that initially reside at one presynaptic terminal suggest that vesicle traffic is not only limited to exchanges with immediate synaptic neighbors, but that individual vesicles can also be shared across multiple terminals (Staras et al., 2010) (Figure 1B). The implications of this are considered later.

\section{CHARACTERISTICS OF MOVING VESICLES}

Experimental evidence, much of it based on the use of FM-dyes fluorescent markers of recycling vesicles (Betz and Bewick, 1992; Ryan et al., 1993) - has provided important insights into functional and organizational characteristics of mobile vesicles. Trafficking vesicles are readily observed in time-lapse sequences of FM-dye labeled neurons (Krueger et al., 2003; Darcy et al., 2006a; Chen et al., 2008; Staras et al., 2010), implying that they must have recently participated in exo-endocytosis. Nonetheless, the relationship between shared vesicles and the endocytic pathways from which they arise remains unknown. In hippocampal neurons, experimental evidence points to multiple modes of vesicle recycling, from fast kiss-and-run fusion (Aravanis et al., 2003; Gandhi and Stevens, 2003) to slower clathrin-mediated endocytosis (Granseth et al., 2006) or endocytosis via intermediate endosomes (Takei et al., 1996). The prevalence of these different pathways is still a subject

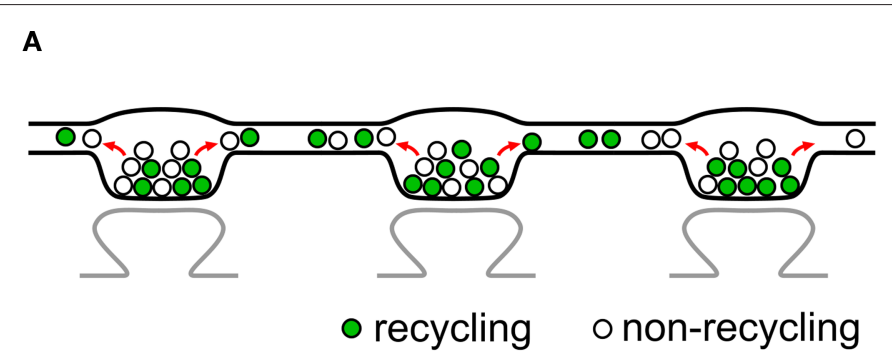

C

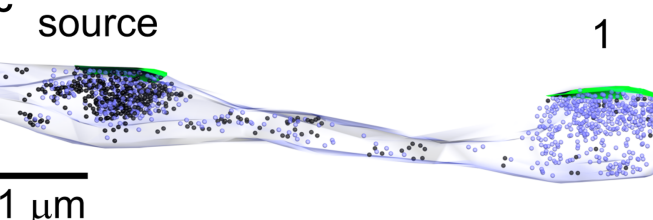

B

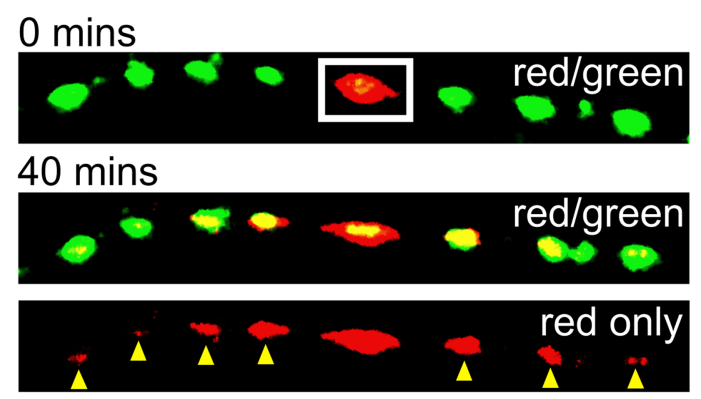

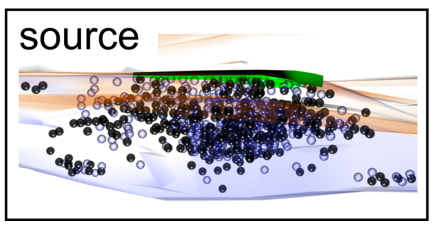

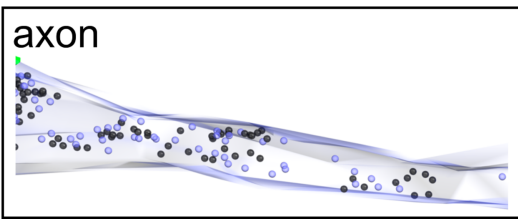

2

3
FIGURE 1 | Sharing of vesicles across multiple synapses. (A) The organization of synapses in hippocampal neurons. Presynaptic terminals are typically formed en passant and each one contains approximately equal numbers of recycling and non-recycling vesicles. Mobile vesicles move both retrogradely and anterogradely between terminals along shared lengths of axons. (B) Vesicle sharing monitored using a photoswitchable vesicle marker, synaptophysinl-Dendra2 (Sypl-Dendra2). Top panel, red/green overlay showing a synapse along an axon being selectively photoswitched (white rectangle) from a green-emitting to a red-emitting form. Middle panel, the spread of red vesicles to neighboring green synapses is monitored over time for up to $40 \mathrm{~min}$. Bottom panel, same image showing red fluorescence only, detailing the contribution of vesicles made from a target synapse to neighbors (yellow arrowheads).
(C) Ultrastructural reconstruction of a hippocampal axon from serial sections, illustrating the lateral trafficking of recycling vesicles from a source synapse to synaptic neighbors. Top, in this experiment samples were photoconverted so that FM-dye-labeled recycling vesicles appear dark. Synapses 1, 2 and 3 were photobleached immediately after labeling to prevent photoconverted product formation, so that all dark vesicles originate from the non-bleached source synapse. The sample was left for 5 min before fixing so that the distribution of photoconverted vesicles reflects lateral sharing from the source synapse. The number of photoconverted vesicles present accumulating at a synapse is related to the distance of that synapse from the source terminal. Bottom, expanded images showing detail from top panel. (B) and (C) are adapted from Staras et al. (2010) with permission from Elsevier. 
of some debate (e.g., Murthy and Stevens, 1998; Granseth et al., 2006; Zhang et al., 2009) but in principle, vesicles that are laterally mobile might be a product of all or just one of these recycling modes. Current evidence does not provide definitive information on this but remains an important issue for future experiments to address. Once they have undergone endocytosis and mobilization, an interesting property of trafficking vesicles is that their fusion competence is retained or at least rapidly acquired after moving into a new synaptic host, indicated by stimulus-evoked FM-dyeloss of newly incorporated fluorescent signal (Darcy et al., 2006a; Staras et al., 2010). Importantly, this functional integration into host presynaptic terminals is also accompanied by spatial integration, with correlative ultrastructural experiments showing that newly incorporated mobile vesicles become readily mixed within the native vesicle cluster (Darcy et al., 2006a) (see Figure 1C).

In what form and by what mechanisms are vesicles conveyed along axons? Ultrastructural analysis (Figure 1C) shows that although some mobile vesicles travel as large clusters, others move in less defined groups or even as single vesicles (Darcy et al., 2006a; Staras et al., 2010) and are functionally heterogeneous with both recycling and non-recycling vesicles present in traveling vesicle packets (Darcy et al., 2006a). Intriguingly, evidence suggests that mobile vesicle clusters can often be fusion-competent during transit (Krueger et al., 2003; Staras et al., 2010) implying that the basic requirements for vesicle fusion can seemingly be met at non-specialized sites along axons. This raises interesting questions about the minimal molecular machinery required to achieve vesicle fusion and the possible functional consequences of extrasynaptic release (see Krueger et al., 2003 for further discussion). Studies using pharmacological blockers implicate microtubules in vesicle movement (Westphal et al., 2008) and reported mobility speeds (Krueger et al., 2003; Darcy et al., 2006a; Westphal et al., 2008) are consistent with transport on microtubule based motors (Brown, 2003). Actin is also implicated in this process, with actin-stabilizing and disrupting agents inhibiting movement of SVs (Darcy et al., 2006a; Westphal et al., 2008) and slowing the accumulation of traveling vesicles at stable synapses (Darcy et al., 2006a). Although these findings are consistent with active transport of vesicles, diffusive movement may also contribute to overall vesicle mobility (Westphal et al., 2008).

Could vesicle sharing in nominally mature neurons simply be a remnant of the vesicle mobility which is associated with developmental processes (Matteoli et al., 1992; Ahmari et al., 2000; Hopf et al., 2002; Sabo et al., 2006)? Several lines of evidence suggest that this is probably not the case. Experiments based on activity-evoked FM-dye loss kinetics indicate that vesicle fusion at extrasynaptic sites in mature neurons has pharmacological sensitivity consistent with mature molecular machinery rather than developmental SV recycling (Krueger et al., 2003). Also, recent evidence from two-photon FM-dye imaging experiments in acute hippocampal rat slices reveals vesicle trafficking events that are broadly analogous to SV movement in cultured neurons (Staras et al., 2010). Indirect evidence in support of extrasynaptic vesicle mobility in mature systems also comes from serial ultrastructural reconstructions of CA3 axons from acute adult hippocampal slice (Shepherd and Harris, 1998). These reveal the presence of synaptic vesicle clusters at intersynaptic sites distinct from presynaptic terminals, analogous to ultrastructural findings in culture (Staras et al., 2010), which could be indicative of mobility of vesicles between synapses in adult brain.

\section{A SHARED VESICLE "SUPERPOOL"}

The existence of functional SV s that are not limited to the boundaries of a presynaptic terminal provides a novel perspective on synaptic organization. The fact that vesicles arising from one synapse are shared with many terminals, taken together with the assumption that multiple and perhaps all terminals contribute vesicles, is consistent with the notion of a synapse-spanning "superpool", a term first coined by (Westphal et al., 2008) and supported by recent work (Staras et al., 2010). How is this new pool defined, and what is its relationship with the classic functionally-defined populations of vesicles (e.g., the recycling pool, readily-releasable pool (RRP) and non-recycling or resting pools) that reside at presynaptic terminals? We view the superpool as an entity which includes all vesicles that appear to be readily available for sharing. In hippocampal terminals, the fraction of recycling vesicles within the total vesicle cluster is highly variable (Harata et al., 2001; Fernandez-Alfonso and Ryan, 2008; Branco et al., 2010), but averages around 45\% (Branco et al., 2010). Of these only a subset - less than half (Staras et al., 2010) appear to be readily mobile and therefore members of the shared pool. It is unclear whether this mobile recycling vesicle component also includes vesicles originating from the RRP. Evidence suggests that non-recycling or resting vesicles are also readily shared (Darcy et al., 2006a; Fernandez-Alfonso and Ryan, 2008). Notably, vesicle composition analysis suggests an approximate correspondence between recycling: non-recycling fractions in extrasynaptic vesicle packets versus those at presynaptic terminals, perhaps indicative of a non-selective recruitment of vesicles into mobile packets (Darcy et al., 2006a). This would be broadly consistent with a simple stochastic model in which selection of vesicles for movement into axons depends on variables such as the number of available "slots" in transport machinery and the proximity of vesicles to this machinery. Thus, the superpool appears to intersect the vesicle population of other pools, existing in a dynamic state where vesicles are constantly interchanged.

A vesicle pool that spans multiple synapses could have consequences for various aspects of synaptic performance, especially given that vesicles appear to have or readily achieve fusion competence upon activity (Krueger et al., 2003; Darcy et al., 2006b; Staras et al., 2010). One possibility is that mobile functional vesicles could act as an additional vesicle reservoir for synaptic release. Time-lapse imaging reveals that fluorescent vesicles can move into presynaptic terminals during the course of sustained stimulation and destain as part of the native vesicle pool (Darcy et al., 2006b; Staras et al., 2010). This implies that mobile vesicles can be available to synapses as an additional functional reserve, extending the conventional boundaries of the synapse vesicle pool and providing another potential variable for dynamic regulation of presynaptic properties. However, whether this is an active and regulated process or simply a consequence of non-regulated vesicle flux whereby vesicles ultimately reach an active synapse during sustained transmission, remains unclear. Another unanswered issue relates to the eventual fate of these vesicles once the synapse returns to a resting state. For example, are they preferentially mobilized or alternatively do they become stable elements of the host vesicle cluster? 
Vesicle sharing could also influence synaptic properties by other means. For example, the observation that vesicle populations at synapses can have different release modes (Goda and Stevens, 1994; Sara et al., 2005; Sun et al., 2007; Fredj and Burrone, 2009) might be indicative of functional heterogeneity among individual vesicles (Voglmaier and Edwards, 2007). In this case, the performance of a synapse receiving mobile vesicles might be altered not only by the number of vesicles being shared but also by the individual and particular molecular properties of each vesicle as bestowed by their synapse of origin. Such a capability for individual vesicles to retain and carry specific functional signatures to new synaptic hosts would presumably depend on the predominant mode of recycling used to retrieve vesicles that were destined to be mobile. For example, while kiss-and-run and clathrin-mediated endocytosis could be compatible with the preservation of individual and specific molecular identities, pathways involving recycling endosomes such as in bulk endocytosis, might serve to "reset" vesicle identity. This interesting issue remains to be addressed.

A further potential consequence of pools of extrasynaptic vesicles is that they could contribute to the regulation of synaptic strength through remodeling of vesicle pools at synapses. It is well-established that release probability, a determinant of synaptic strength (Branco and Staras, 2009), is correlated with recycling vesicle pool size (Murthy et al., 1997). This implies that changes in the mechanisms controlling vesicle release/capture and leading to increases or decreases in functional pool sizes could impact on synaptic efficacy (Staras, 2007). Indeed, if vesicles were drawn locally from a large and readily accessible extrasynaptic pool which is continuously replenished, consistent with the properties of the superpool, the resizing of the vesicle pool at one presynaptic terminal might be achieved relatively quickly. Importantly, this could happen without significant effects on synaptic neighbors, allowing independent regulation of individual synapses. This type of mechanism for adjusting synaptic weights could thus contribute to a variety of different forms of plasticity, from the synapse-specific homeostatic changes in recycling pool sizes and release probability (Murthy et al., 2001; Thiagarajan et al., 2005; Branco et al., 2008; Branco and Staras, 2009) to the resizing of synaptic pools associated with forms of potentiation (Ninan et al., 2006; Tyler et al., 2006). It could also contribute to the established synapse-synapse variability in release probability (Branco et al., 2010) providing a means for rapid redistribution of presynaptic weights. Directed recruitment from, or release of vesicles to, the superpool could also support forms of synaptogenesis or synapse elimination, known to occur over time in vivo (De Paola et al., 2006; Stettler et al., 2006) and also likely to accompany some activity-dependent forms of plasticity (Antonova et al., 2001; Colicos et al., 2001; Nikonenko et al., 2003).

At present, the regulatory mechanisms that might control the release/capture of vesicles at individual terminals to support modulation of synaptic performance are not known. Clues to possible functional mechanisms might be offered by other systems. In Drosophila neuromuscular junction, elegant work has revealed that sustained peptidergic transmission relies on continuous trafficking of large-dense core vesicles through synaptic junctions from the soma (Shakiryanova et al., 2006). To permit targeted refilling, vesicle capture mechanisms involving a presynaptic ryanodine receptor-CamKII signaling pathway (Wong et al., 2009) are engaged at activated synapses, allowing new vesicles to be recruited for release. This provides an important illustration of how local capture machinery can permit a presynaptic terminal to utilize extrasynaptic vesicles to meet synaptic demand. In hippocampal neurons, one possible candidate for the regulation of vesicle clustering is brain-derived neurotrophic factor (BDNF), operating via cadherin-catenin adhesion complexes through activation of TrkB receptors. Global BDNF application can lead to transient liberation of vesicles from synapses (Bamji et al., 2006) and with focal application, this vesicle declustering can occur at a local level (Staras et al., 2010), providing a possible synapse-specific control mechanism for presynaptic vesicle organization. Notably, BDNF has been implicated in presynaptic expression of long-term potentiation (Zakharenko et al., 2003; Tyler et al., 2006), suggesting it could be a pivotal element in regulating presynaptic remodeling associated with forms of long-term plasticity.

\section{SYNAPTIC COMPONENTS AND LATERAL MOBILITY: A CONSENSUS THEME}

The finding that SVs are readily shared across multiple terminals is consistent with a growing literature on the mobility of other nonvesicular synaptic components. Transient, localized redistribution, which is typically linked to SV recycling processes, has been reported for a variety of elements including clathrin (Mueller et al., 2004; Granseth et al., 2006), Rab3 (Star et al., 2005), synaptobrevin (Li and Murthy, 2001; Granseth et al., 2006) and N-cadherin (Tanaka et al., 2000). But longer-range constitutive exchange of synaptic components, sharing dynamic features with the movement of vesicles, has also been established. Synapsin, for example, is highly mobile, redistributing continuously among synapses with a timescale of minutes to hours (Tsuriel et al., 2006). Similar trafficking dynamics have also been identified for Munc13-1, an important active zone protein (Kalla et al., 2006). These findings on dynamic movement of presynaptic components are more than matched by studies establishing lateral trafficking of postsynaptic elements (Jaskolski and Henley, 2009). These include key components of the postsynaptic density such as $\mathrm{Ca}^{2+}$ /calmodulin-dependent protein kinase II (CaMKII) (Okamoto et al., 2004; Sharma et al., 2006), NMDA NR1 (Sharma et al., 2006), AMPA GluR1 (Sharma et al., 2006; Bats et al., 2007), PSD-95 (Okabe et al., 2001; Gray et al., 2006; Sharma et al., 2006) and ProSAP2 (Tsuriel et al., 2006). Indeed, strong evidence points to a link between forms of synaptic plasticity and regulated capture/release of important postsynaptic elements (Sharma et al., 2006; Petrini et al., 2009). Taken together, these findings point to an emerging consensus about the dynamic nature of synapse-related traffic. New studies are now required to simultaneously monitor the dynamics of pre and postsynaptic elements and establish their relationship during trafficking processes.

At first glance the notion of highly dynamic synaptic components seems somewhat incompatible with the idea that synapses endure over time (De Paola et al., 2006; Stettler et al., 2006). How do synapses maintain an organizational and functional identity in the face of large-scale sharing of synaptic components - an important demand to ensure transmission of the correct message to the right target. For SVs, a partial answer to this question might relate to the fact that vesicle sharing appears to be limited to only a subset $(\sim 40 \%)$ of functional vesicles that reside at a synapse (Staras et al., 2010). In this way, significant numbers of vesicles could contribute to a 
mobile pool, but the structural and functional integrity of a synapse might still be preserved by the maintenance of a stable vesicle "core". Also, although minute-to-minute fluctuations in the trafficking of vesicles through synapses are observed, the presynaptic terminal maintains a relatively constant average vesicle pool size over longer time periods (Darcy et al., 2006a; Staras et al., 2010), suggesting that the persistence of a cluster of vesicles and specificity of synaptic function might be achieved by the relatively tight regulation of vesicle capture/release. Preservation of synaptic specificity might also be conferred by key structural elements of the synapse. Indeed, the apparent importance of these elements becomes clear when a pivotal element such as the cadherin-catenin adhesion complex is disrupted, leading to SV declustering and presynaptic disassembly (Bamji et al., 2003, 2006; Staras et al., 2010). Elegant work by Tsuriel et al. (2009), examining the structural active zone component, Bassoon, illustrates how this component is stable over a period of hours (Tsuriel et al., 2009) suggesting this and other proteins alike may also be key elements in the maintenance of synaptic integrity (see Garner et al., 2006 for further discussion).

\section{REFERENCES}

Ahmari, S. E., Buchanan, J., and Smith, S. J. (2000). Assembly of presynaptic active zones from cytoplasmic transport packets. Nat. Neurosci. 3, 445-451.

Antonova, I., Arancio, O., Trillat, A. C., Wang, H. G., Zablow, L., Udo, H., Kandel, E. R., and Hawkins, R. D. (2001). Rapid increase in clusters of presynaptic proteins at onset of longlasting potentiation. Science 294, 1547-1550.

Aravanis, A. M., Pyle, J. L., and Tsien, R. W. (2003). Single synaptic vesicles fusing transiently and successively without loss of identity. Nature 423, 643-647.

Bamji, S. X., Rico, B., Kimes, N., and Reichardt, L. F. (2006). BDNF mobilizes synaptic vesicles and enhances synapse formation by disrupting cadherin-beta-catenin interactions. J. Cell Biol. 174, 289-299.

Bamji, S. X., Shimazu, K., Kimes, N., Huelsken, J., Birchmeier, W., Lu, B., and Reichardt, L. F. (2003). Role of beta-catenin in synaptic vesicle localization and presynaptic assembly. Neuron 40, 719-731.

Bats, C., Groc, L., and Choquet, D. (2007). The interaction between Stargazin and PSD-95 regulates AMPA receptor surface trafficking. Neuron 53, 719-734.

Betz, W. J., and Bewick, G. S. (1992). Optical analysis of synaptic vesicle recycling at the frog neuromuscular junction. Science 255, 200-203.

Branco, T., Marra, V., and Staras, K. (2010). Examining size-strength relationships at hippocampal synapses using an ultrastructural measurement of synaptic release probability. J. Struct. Biol. http://dx.doi.org/10.1016/j. jsb.2009.10.014.

Branco, T., and Staras, K. (2009). The probability of neurotransmitter control at single synapses. Nat. Rev. Neurosci. 10, 373-383.

Branco, T., Staras, K., Darcy, K. J., and Goda, Y. (2008). Local dendritic activity sets release probability at hippocampal synapses. Neuron 59, 475-485.

Brown, A. (2003). Axonal transport of membranous and nonmembranous cargoes: a unified perspective. J. Cell Biol. 160, 817-821.

Ceccarelli, B., Hurlbut, W. P., and Mauro, A. (1973). Turnover of transmitter and synaptic vesicles at the frog neuromuscular junction. J. Cell Biol. 57, 499-524.

Chen, X., Barg, S., and Almers, W. (2008). Release of the styryl dyes from single synaptic vesicles in hippocampal neurons. J. Neurosci. 28, 1894-1903.

Colicos, M. A., Collins, B. E., Sailor, M. J., and Goda, Y. (2001). Remodeling of synaptic actin induced by photoconductive stimulation. Cell 107, 605-616.

Darcy, K. J., Staras, K., Collinson, L. M., and Goda, Y. (2006a). Constitutive sharing of recycling synaptic vesicles between presynaptic boutons. Nat. Neurosci. 9, 315-321.

Darcy, K. J., Staras, K., Collinson, L. M., and Goda, Y. (2006b). An ultrastructural readout of fluorescence recovery after photobleaching using correlative light and electron microscopy. Nat. Protoc. 1, 988-994.

De Paola, V., Holtmaat, A., Knott, G., Song, S., Wilbrecht, L., Caroni, P., and Svoboda, K. (2006). Cell type-specific structural plasticity of axonal branches and boutons in the adult neocortex. Neuron 49, 861-875.

De Robertis, E. D., and Bennett, H. S. (1954). A submicroscopic vesicular release: variability and feedback

\section{CONCLUSIONS}

In hippocampal neurons, populations of SVs are highly dynamic, crossing the conventional boundaries of a presynaptic terminal and being shared readily across multiple synaptic boutons. This finding represents a novel view of presynaptic organization in which shared vesicles can impact on synaptic function and where the regulation of vesicle traffic could be relevant to the control and modulation of synaptic properties. It is also consistent with a burgeoning literature establishing the importance of regulated movement of other non-vesicular synaptic components, both pre- and postsynaptically, and the consequences of this for synaptic function. Experiments detailing the pathways that control sharing of SVs and the conditions under which vesicle flows are adjusted, both in hippocampal neurons and other systems, will be key issues for future work to address.

\section{ACKNOWLEDGMENTS}

Kevin Staras is supported by Wellcome Trust (WT084357MF) and BBSRC (BB/F018371) grants. Tiago Branco is supported by the Gatsby Charitable Foundation.

component of Schwann cells and nerve satellite cells. Exp. Cell Res. 6, 543-545.

De Robertis, E. D., and Bennett, H. S. (1955). Some features of the submicroscopic morphology of synapses in frog and earthworm. J. Biophys. Biochem. Cytol. 1, 47-58.

Fernandez-Alfonso, T., and Ryan, T. A. (2006). The efficiency of the synaptic vesicle cycle at central nervous system synapses. Trends Cell Biol. 16, 413-420.

Fernandez-Alfonso, T., and Ryan, T. A. (2008).A heterogeneous "resting" pool of synaptic vesicles that is dynamically interchanged across boutons in mammalian CNS synapses. Brain Cell Biol. 36, 87-100.

Fredj, N. B., and Burrone, J. (2009). A resting pool of vesicles is responsible for spontaneous vesicle fusion at the synapse. Nat. Neurosci. 12, 751-758.

Gandhi, S. P., and Stevens, C. F. (2003). Three modes of synaptic vesicular recycling revealed by single-vesicle imaging. Nature 423, 607-613.

Garner, C. C., Waites, C. L., and Ziv, N. E. (2006). Synapse development: still looking for the forest, still lost in the trees. Cell Tissue Res. 326, 249-262.

Goda, Y., and Stevens, C. F. (1994). Two components of transmitter release at a central synapse. Proc. Natl. Acad. Sci. U.S.A. 91, 12942-12946.

Granseth, B., Odermatt, B., Royle, S. J., and Lagnado, L. (2006). Clathrin-mediated endocytosis is the dominant mechanism of vesicle retrieval at hippocampal synapses. Neuron 51, 773-786.

Gray, N. W., Weimer, R. M., Bureau, I., and Svoboda, K. (2006). Rapid redistribution of synaptic PSD-95 in the neocortex in vivo. PLoS Biol. 4, e370. doi: 10.1371/journal.pbio.0040370.
Harata, N., Ryan, T. A., Smith, S. J., Buchanan, J., and Tsien, R. W. (2001). Visualizing recycling synaptic vesicles in hippocampal neurons by FM 1-43 photoconversion. Proc. Natl. Acad. Sci. U.S.A. 98, 12748-12753.

Heuser, J. E., and Reese, T. S. (1973). Evidence for recycling of synaptic vesicle membrane during transmitter release at the frog neuromuscular junction. J. Cell Biol. 57, 315-344.

Hopf, F. W., Waters, J., Mehta, S., and Smith, S. J. (2002). Stability and plasticity of developing synapses in hippocampal neuronal cultures. $J$. Neurosci. 22, 775-781.

Ishizuka, N., Weber, J., and Amaral, D. G. (1990). Organization of intrahippocampal projections originating from CA3 pyramidal cells in the rat. J. Comp. Neurol. 295, 580-623.

Jaskolski, F., and Henley, J. M. (2009). Synaptic receptor trafficking: the lateral point of view. Neuroscience 158, 19-24.

Jordan, R., Lemke, E. A., and Klingauf, J. (2005). Visualization of synaptic vesicle movement in intact synaptic boutons using fluorescence fluctuation spectroscopy. Biophys. J. 89, 2091-2102.

Kalla, S., Stern, M., Basu, J., Varoqueaux, F., Reim,K., Rosenmund,C.,Ziv, N.E., and Brose, N. (2006). Molecular dynamics of a presynaptic active zone protein studied in Munc13-1-enhanced yellow fluorescent protein knock-in mutant mice. J. Neurosci. 26, 13054-13066.

Kraszewski, K., Daniell, L., Mundigl, O., and De Camilli, P. (1996). Mobility of synaptic vesicles in nerve endings monitored by recovery from photobleaching of synaptic vesicleassociated fluorescence. J. Neurosci. 16, 5905-5913. 
Krueger, S. R., Kolar, A., and Fitzsimonds, R. M. (2003). The presynaptic release apparatus is functional in the absence of dendritic contact and highly mobile within isolated axons. Neuron 40, 945-957.

Lemke, E. A., and Klingauf, J. (2005). Single synaptic vesicle tracking in individual hippocampal boutons at rest and during synaptic activity. $J$. Neurosci. 25, 11034-11044.

Li,Z., and Murthy, V.N. (2001).Visualizing postendocytic traffic of synaptic vesicles at hippocampal synapses. Neuron 31, 593-605.

Matteoli, M., Takei, K., Perin, M. S., Sudhof, T. C., and De Camilli, P. (1992). Exo-endocytotic recycling of synaptic vesicles in developing processes of cultured hippocampal neurons. J. Cell Biol. 117, 849-861.

Mueller, V. J., Wienisch, M., Nehring, R. B., and Klingauf, J. (2004). Monitoring clathrin-mediated endocytosis during synaptic activity. J. Neurosci. 24, 2004-2012.

Murthy, V. N., Schikorski, T., Stevens, C. F., and Zhu, Y. (2001). Inactivity produces increases in neurotransmitter release and synapse size. Neuron 32, 673-682.

Murthy, V.N., Sejnowski, T. J., and Stevens, C. F. (1997). Heterogeneous release properties of visualized individual hippocampal synapses. Neuron 18, 599-612.

Murthy, V. N., and Stevens, C. F. (1998). Synaptic vesicles retain their identity through the endocytic cycle. Nature 392, 497-501.

Nikonenko, I., Jourdain, P., and Muller, D. (2003). Presynaptic remodeling contributes to activity-dependent synaptogenesis. J. Neurosci. 23, 8498-8505.

Ninan, I., Liu, S., Rabinowitz, D., and Arancio, O. (2006). Early presynaptic changes during plasticity in cultured hippocampal neurons. EMBO J. 25, 4361-4371.

Okabe, S., Urushido, T., Konno, D., Okado, H., and Sobue, K. (2001). Rapid redistribution of the postsynaptic density protein PSD-Zip45 (Homer 1c) and its differential regulation by NMDA receptors and calcium channels. J. Neurosci. 21, 9561-9571.

Okamoto, K., Nagai, T., Miyawaki, A., and Hayashi, Y. (2004). Rapid and persistent modulation of actin dynamics regulates postsynaptic reorganization underlying bidirectional plasticity. Nat. Neurosci. 7, 1104-1112.
Palay, S. L. (1956). Synapses in the central nervous system. J. Biophys. Biochem. Cytol. 2, 193-202.

Petrini, E. M., Lu, J., Cognet, L., Lounis, B., Ehlers, M.D., and Choquet, D. (2009). Endocytic trafficking and recycling maintain a pool of mobile surface AMPA receptors required for synaptic potentiation. Neuron 63, 92-105.

Rizzoli, S. O., and Betz, W. J. (2005). Synaptic vesicle pools. Nat. Rev. Neurosci. 6, 57-69.

Ryan, T. A. (2001). Presynaptic imaging techniques. Curr. Opin. Neurobiol. 11, 544-549.

Ryan, T. A., Reuter, H., Wendland, B., Schweizer, F. E., Tsien, R. W., and Smith, S. J. (1993). The kinetics of synaptic vesicle recycling measured at single presynaptic boutons. Neuron 11, 713-724

Ryan, T. A., Smith, S. J., and Reuter, H. (1996). The timing of synaptic vesicle endocytosis. Proc. Natl. Acad. Sci. U.S.A. 93, 5567-5571.

Sabo, S. L., Gomes, R. A., and McAllister, A. K. (2006). Formation of presynaptic terminals at predefined sites along axons. J. Neurosci. 26, 10813-10825.

Sara, Y., Virmani, T., Deak, F., Liu, X., and Kavalali, E. T. (2005). An isolated pool of vesicles recycles at rest and drives spontaneous neurotransmission. Neuron 45, 563-573.

Schweizer, F. E., and Ryan, T. A. (2006). The synaptic vesicle: cycle of exocytosis and endocytosis. Curr. Opin. Neurobiol. 16, 298-304.

Shakiryanova, D., Tully, A., and Levitan, E. S. (2006). Activity-dependent synaptic capture of transiting peptidergic vesicles. Nat. Neurosci. 9, 896-900.

Sharma, K., Fong, D. K., and Craig, A. M. (2006). Postsynaptic protein mobility in dendritic spines: long-term regulation by synaptic NMDA receptor activation. Mol. Cell. Neurosci. 31, 702-712.

Shepherd, G. M., and Harris, K. M. (1998). Three-dimensional structure and composition of $\mathrm{CA} 3 \rightarrow \mathrm{CA} 1$ axons in rat hippocampal slices: implications for presynaptic connectivity and compartmentalization. J. Neurosci. $18,8300-8310$.

Shtrahman, M., Yeung, C., Nauen, D. W., Bi, G. Q., and Wu, X. L. (2005). Probing vesicle dynamics in single hippocampal synapses. Biophys. J. 89, 3615-3627.

Sorra, K. E., and Harris, K. M. (1993). Occurrence and three-dimensional structure of multiple synapses between individual radiatum axons and their target pyramidal cells in hippocampal area CA1. J. Neurosci. 13, 3736-3748.

Star, E. N., Newton, A. J., and Murthy, V. N. (2005). Real-time imaging of Rab3a and Rab5a reveals differential roles in presynaptic function. J. Physiol. (Lond.) 569, 103-117.

Staras, K. (2007). Share and share alike: trading of presynaptic elements between central synapses. Trends Neurosci. 30, 292-298.

Staras, K., Branco, T., Burden, J. J., Pozo, K., Darcy, K. J., Marra, V., Ratnayaka, A., and Goda, Y. (2010). A vesicle superpool spans multiple presynaptic terminals in hippocampal neurons. Neuron 66, 37-44.

Stettler, D. D., Yamahachi, H., Li, W., Denk, W., and Gilbert, C. D. (2006). Axons and synaptic boutons are highly dynamic in adult visual cortex. Neuron 49, 877-887.

Sudhof, T. C. (2004). The synaptic vesicle cycle. Annu. Rev. Neurosci. 27, 509-547.

Sun, J., Pang, Z. P., Qin, D., Fahim, A. T., Adachi, R., and Sudhof, T. C. (2007). A dual-Ca2+-sensor model for neurotransmitter release in a central synapse. Nature 450, 676-682.

Tanaka, H., Shan, W., Phillips, G. R., Arndt, K., Bozdagi, O., Shapiro, L., Huntley, G. W., Benson, D. L., and Colman, D. R. (2000). Molecular modification of $\mathrm{N}$-cadherin in response to synaptic activity. Neuron 25, 93-107.

Takei, K., Mundigl, O., Daniell, L., and De Camilli, P. (1996). The synaptic vesicle cycle: a single vesicle budding step involving clathrin and dynamin. J. Cell Biol. 133, 1237-1250.

Thiagarajan, T. C., Lindskog, M., and Tsien, R. W. (2005). Adaptation to synaptic inactivity in hippocampal neurons. Neuron 47, 725-737.

Tsuriel, S., Fisher, A., Wittenmayer, N., Dresbach, T., Garner, C. C., and Ziv, N.E. (2009). Exchange and redistribution dynamics of the cytoskeleton of the active zone molecule bassoon. $J$. Neurosci. 29, 351-358.

Tsuriel, S., Geva, R., Zamorano, P., Dresbach, T., Boeckers, T., Gundelfinger,E.D., Garner, C.C., and Ziv, N. E. (2006). Local sharing as a predominant determinant of synaptic matrix molecular dynamics. PLoS Biol. 4, e271. doi: 10.1371/journal. pbio.0040271.

Tyler, W. J., Zhang, X. L., Hartman, K., Winterer, J., Muller, W., Stanton, P. K., and Pozzo-Miller, L. (2006).
BDNF increases release probability and the size of a rapidly recycling vesicle pool within rat hippocampal excitatory synapses. J. Physiol. (Lond.) 574, 787-803.

Voglmaier, S. M., and Edwards, R. H. (2007). Do different endocytic pathways make different synaptic vesicles? Curr. Opin. Neurobiol. 17, 374-380.

Westphal, V., Rizzoli, S. O., Lauterbach, M. A., Kamin, D., Jahn, R., and Hell, S W. (2008). Video-rate far-field optical nanoscopy dissects synaptic vesicle movement. Science 320, 246-249.

Westrum, L. E., and Blackstad, T. W. (1962).An electron microscopic study of the stratum radiatum of the rat hippocampus (regio superior, CA 1) with particular emphasis on synaptology. J. Comp. Neurol. 119, 281-309.

Wong, M. Y., Shakiryanova, D., and Levitan, E. S. (2009). Presynaptic ryanodine receptor-CamKII signaling is required for activity-dependent capture of transiting vesicles. J. Mol. Neurosci. 37, 146-150.

Zakharenko, S. S., Patterson, S. L., Dragatsis, I., Zeitlin, S. O., Siegelbaum, S. A., Kandel, E. R., and Morozov, A. (2003). Presynaptic BDNF required for a presynaptic but not postsynaptic component of LTP at hippocampal CA1-CA3 synapses. Neuron 39, 975-990.

Zhang, Q., Li, Y., and Tsien, R. W. (2009). The dynamic control of kiss-andrun and vesicular reuse probed with single nanoparticles. Science 323, 1448-1453.

Conflict of Interest Statement: The authors declare that the research was conducted in the absence of any commercial or financial relationships that could be construed as a potential conflict of interest.

Received: 27 April 2010; paper pending published: 18 May 2010; accepted: 27 May 2010; published online: 17 June 2010. Citation: Staras K and Branco T (2010) Sharing vesicles between central presynaptic terminals: implications for synaptic function. Front. Syn. Neurosci. 2:20. doi: 10.3389/fnsyn.2010.00020

Copyright (c) 2010 Staras and Branco. This is an open-access article subject to an exclusive license agreement between the authors and the Frontiers Research Foundation, which permits unrestricted use, distribution, and reproduction in any medium, provided the original authors and source are credited. 